TFIIE and - $\mathrm{J}$ do remains undefined. Pol II that lacks a CTD can still initiate transcription in vitro, which may indicate that phosphorylation of the CTD is only one component of the trigger that uncouples Pol II from the preinitiation complex.

Moreover, the establishment of TFIIH as a specific CTD kinase does not rule out the possibility that other kinases may be involved in transcription initiation or even post-initiation events. For example, the cell-cycle regulating kinase, M-phase promoting factor, also phosphorylates the CTD, perhaps at different residues to those phosphorylated by TFIIH. Intriguingly, this phosphorylated form of Pol II is actually abrogated for transcription. So other kinases may target different residues of the Pol II CTD to modulate transcription by distinct signalling mechanisms, whereas TFIIH appears to serve as part of the switch to kick RNA polymerase into the actively transcribing form of the enzyme.

The remaining five general transcription factors, many of them multiprotein complexes, possess activities that are yet to be defined. What their functions turn out to be, as we unravel the mechanisms of transcription initiation, are tales to be told another day.

Michael Gregory Peterson is at Tularik Inc., 270 East Grand Avenue, South San Francisco, California 94080, USA. Robert Tjian is in the Howard Hughes Medical Institute, Department of Molecular and Cell Biology, University of California, Berkeley, California 94720, USA.

\title{
Towards energy storage
}

\section{Prabir Dutta}

EFFICIENT solar energy storage may become possible following work described by Vermeulen and Thompson on page 656 of this issue ${ }^{l}$. The authors describe a halide-viologen complex trapped in an inorganic matrix which can store solar optical energy indefinitely.

The conversion of solar energy into storable electrical or chemical energy would make an attractive alternative to the burning of fossil fuels. Photovoltaic systems currently in use are a step along this direction, and great strides are being made in improving these and other systems based on photoelectrochemistry ${ }^{2,3}$. One strategy commonly adapted in work on permanent storage is to use lightinduced separation of charge in electron donor-acceptor compounds. The redox products, in principle, can then be used in subsequent reactions to release the stored energy. The success of this strategy, however, depends on being able to thwart the back reaction between the redox products.

In their work, Vermeulen and Thompson use a halide-viologen unit for charge separation. The blue colour of the viologen radical formed in the photoredox reaction persists indefinitely, even in the presence of air. Evidently, this arises from the arrangement of the complex in the interlayer space of a zirconium phosphonate matrix - in homogeneous solution, the back electron transfer is facile.

Those in the field, taking their cue from natural photosynthetic systems, have long recognized that a precise and ordered assembly of molecules is required for effective photoinduced charge separation. Various strategies are being NATURE · VOL358 - 20 AUGUST 1992 developed. These range from elegant syntheses of donor and acceptor molecules held at specific distances and geometries by spacer groups ${ }^{4,5}$, to microheterogeneous systems that promote the optimal assembly of donor and acceptor species $^{6}$. Vermeulen and Thompson's report is an example of the latter category and compares well with other matrices, such as zeolites ${ }^{7,8}$ and sol-gel glasses ${ }^{9}$. In these supports, the cages and cavities promote optimal alignment as well as mobility to ensure long-lived charge separation. In Vermeulen and Thompson's example, the alignment of the interlayer viologens promotes electron delocalization over many viologen molecules. Besides, a unique feature of their system that may be responsible for the slow back electron transfer reaction is the possibility that the framework $\mathrm{ZrO}_{6}$ unit may be donating an electron to the halide radical. Thus, in the case of this novel support, not only are the photoactive molecules being aligned in specific geometries, but the framework itself is participating in the charge transfer process.

There are several aspects of this system that will need to be improved before it becomes a practical energy storage material. First, its photoresponse needs to be moved to longer wavelengths so that a larger fraction of the solar spectrum is absorbed. One way would be to use iodide ions, which give viologen charge-transfer bands that extend beyond $600 \mathrm{~nm}$. Replacement of zirconium by other metals could promote the electron transfer to halide radicals and so increase the charge separation yield. Also, one must be able to extract the

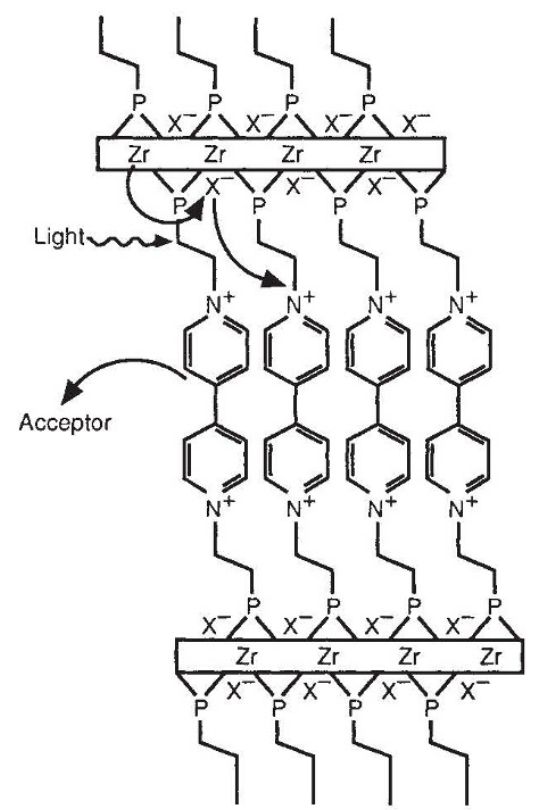

Energy storage and utilization in a viologenzirconium-phosphonate system. Visible light brings about electron transfer from halide $(X-)$ to the viologen moiety. The framework $\mathrm{ZrO}_{6}$ donates an electron to the halide radical, resulting in physical separation of charges and thereby long-lived charge separation. To use the redox equivalent on the viologen, a possible route would be charge propagation followed by electron donation to an appropriate acceptor in solution. The light energy is ultimately used to generate useful chemical products via reactions of the acceptor.

energy stored, in which case the viologen in the interlayer must be accessible. This is a problem because of the matrix's close-packed lattice. One strategy might be to promote charge migration along the packed viologens followed by transfer of the electron from the solid to a species in solution phase whose reduction potential is lower than that of interlamellar viologen (see figure).

Although making these changes, and making them work, will be no trivial matter, the new work clearly shows that it should be possible to make solar energy storage devices and maybe batteries in the not-too-distant future.

Prabir Dutta is in the Department of Chemistry, Ohio State University, Columbus, Ohio 43210-1173, USA.

\footnotetext{
1. Vermeulen, L. A. \& Thompson, M. E. Nature 358 $656-658$ (1992)

2. O'Regan, B. \& Grätzel, M. Nature $353,737-740$ (1991)

3. Nozik, A. Phil. Trans. R. Soc. A295, 453-470 (1980).

4. Gust, D. et al. Science 248, 199-201 (1990)

5. Gaines, G. L. III, O'Neil, M. P., Suec, W. A., Niemczyk, M. P. \& Wasielewski, M. R. J. Am. chem. Soc. 113, 719-721 (1991)

6. Ramamurthy, V. Photochemistry in Organized and Constrained Media (VCH, New York, 1991)

7. Dutta, P. K. \& Incavo, J. J. phys. Chem. 91, 4443 4446 (1987).

8. Krueger, J. S., Mayer, J. E. \& Mallouk, T. E. J. Am. chem. Soc. 110, 8232-8234 (1988).

9. Slama-Schwok, A., Ottolenghi, M. \& Avnir, D. Nature 355, 240-242 (1992)
} 